\title{
Diacronie
}

Studi di Storia Contemporanea

$\mathrm{N}^{\circ} 21,1 \mid 2015$

Le città di Babele

\section{UCEI (a cura di), Migrazioni, Memorie: La Rassegna Mensile di Israel, LXXVIII, 3/2012}

\section{Luca Zuccolo}

\section{Q OpenEdition \\ Journals}

\section{Edizione digitale}

URL: http://journals.openedition.org/diacronie/1930

DOI: $10.4000 /$ diacronie. 1930

ISSN: 2038-0925

\section{Editore}

Association culturelle Diacronie

\section{Notizia bibliografica digitale}

Luca Zuccolo, « UCEI (a cura di), Migrazioni, Memorie: La Rassegna Mensile di Israel, LXXVIII, 3/2012 », Diacronie [Online], $N^{\circ} 21,1 \mid$ 2015, documento 10, Messo online il 01 mars 2015, consultato il 24 septembre 2020. URL : http://journals.openedition.org/diacronie/1930 ; DOI : https://doi.org/10.4000/ diacronie. 1930 


\section{RECENSIONE:}

\section{UCEI (a cura di), Migrazioni, Memorie : La Rassegna Mensile di Israel, LXXVIII, 3/2012, Firenze, La Giuntina, 2013, 181 pp.}

a cura di Luca ZUCCOLO *

«Rivista letta e conosciuta in Europa, Israele e Stati Uniti», come ricorda il sito dell'UCEI, "La Rassegna Mensile di Israel” è divenuta da tempo uno strumento indispensabile allo studioso della storia, del pensiero, della letteratura degli ebrei in Italia». Nato nel 1925, questo quadrimestrale, giunto al volume LXXVIII, «ha continuato la sua pubblicazione con lo scopo di rendere accessibili ai suoi lettori le problematiche religiose, politiche, sociali e storico-culturali dell'ebraismo, dando modo di conoscere anche nuove ricerche in ambito archeologico, letterario, artistico e scientifico ${ }^{1}$. Progetto che viene egregiamente perseguito anche nel numero 3 del settembre-dicembre 2012 dal titolo Migrazioni, Memorie, qui presentato.

Il volume, come ricorda nel suo editoriale Giacomo Saban², raccoglie svariati articoli che trattano dei temi più vari ma che hanno come filo rosso l'identità, o meglio le identità, degli ebrei. Fin dal titolo - declinato al plurale - infatti, si capisce che non unica è l'identità ebraica e nemmeno la sua rappresentazione o il suo ricordo. I contributi del volume sebbene molto diversi tra loro compongono un'unità sfaccettata che racconta storie e memorie delle molteplici comunità ebraiche diasporiche, come di quella israeliana; ma allo stesso tempo il volume è anche espressione di memorie di singoli individui che divengono, nella loro stessa narrazione, parte integrante di una memoria condivisa. Gli articoli, sebbene diversi tra loro, creano un percorso che guida il lettore attraverso una comprensione più chiara di persone e temi storici complessi a

\footnotetext{
${ }^{1} \mathrm{URL}:$

$<$ http://www.ucei.it/default.asp?cat=7\&cattitle=cultura\&pag=84\&pagtitle=rassegna_mensile_ di_israel $>$ [consultato il 21 aprile 2014].

2 SABAN, Giacomo, «Editoriale», in UCEI (a cura di), La Rassegna Mensile di Israel : Migrazioni, Memorie, LXXVIII, 3/ 2012, Firenze, La Giuntina, 2013, p. VII.
} 
cui spesso il pubblico si avvicina con difficoltà e che sono frequentemente fraintesi a causa di una pubblicistica sempre più diffusa e di facile reperibilità che stravolge la realtà e la memoria di un passato doloroso.

Il numero si divide in quattro parti tematiche: Migrazioni, Memorie, Ebrei Italiani, Letterature e Ebraismo nei Territori Ottomani, le quali, indagando storie personali e collettive, raccontano la pluralità della realtà ebraica e la sua complessità e profondità. La prima parte si apre con il saggio di Giuseppe Veltri, Culture Ebraiche e migrazioni del sapere. Tre Tesi, in cui l'autore - professore ordinario di Studi Ebraici all'Università di Halle-Wittenberg, docente onorario all'Università di Lipsia e direttore dell'«European Journal of Jewish Studies» (edito dalla Brill) - propone e presenta tre tesi sulla cultura nel giudaismo e sul rapporto tra migrazione e cultura, sostenendo il ruolo decisivo degli ebrei diasporici nella diffusione di scienze e idee. Ad esso fa seguito l’interessante capitolo di Luca Zevi, Luoghi di una memoria (più o meno) ben temperata che descrive e interpreta le memorie e la loro condivisione. Il saggio, che si apre con la storia umana di Fabio Mauri e della sua vita come metafora della memoria della Shoà, propone una disamina della memoria e delle pratiche memoriali - musei, monumenti - emersi in Europa e nel mondo dopo la tragedia della Shoà mettendo in evidenza non solo lo sforzo e il dolore di ricordare degli eventi così drammatici, ma anche il pericolo, sempre latente, di una spettacolarizzazione e di un'astrazione dell'evento dalla sua realtà contingente; pericolo che renderebbe, secondo l'autore, difficile ogni sforzo mnemonico e pedagogico facendo emergere solo la celebrazione e marginalizzando, invece, la riflessione.

La seconda parte, Ebrei Italiani, sposta il campo dell'analisi dalla comunità ai singoli individui, dalla memoria alla storia, con due saggi che danno spazio alle storie personali di due ebrei italiani a loro modo simbolo di due realtà storiche del nostro Paese poco conosciute. Apre la sezione il saggio di Elizabeth Schächter, Carlo Alberto Viterbo, un protagonista dell'ebraismo italiano, in cui l'autrice descrive la vita e le opere socio-politiche all'interno della comunità ebraica italiana e internazionale di Carlo Viterbo, il quale avvicinatosi al sionismo ne diventa il principale esponente italiano. Calate in un periodo storico tra i più complessi e difficili per gli ebrei - gli anni del fascismo e della guerra - le attività di Viterbo, tra cui spiccano quelle culturali ed editoriali, si presentano come un utile strumento di comprensione e condivisione dell'esperienza ebraica in Italia. Un'esperienza che grazie all'UCII (Unione delle Comunità Israelitiche Italiane) e ad uomini come Viterbo e alla sua opera editoriale hanno permesso di approfondire la conoscenza del mondo degli ebrei italiani. 
Di una comunità ebraica tra le più antiche del nostro paese, invece, parla il saggio di Paola Ferruta, Ricca Luzzatto e la "contaminazione" della giustizia nella Venezia del Seicento, in cui attraverso la storia di Ricca e della sua famiglia, l'autrice fa luce su alcuni aspetti della non sempre facile relazione tra gli ebrei del Ghetto e i nobili della Serenissima Repubblica. La storia familiare e della comunità evidenzia non solo le dinamiche relazionali interne al Ghetto ma soprattutto quelle tra i suoi abitanti e la popolazione di Venezia in un periodo come il Seicento in cui la crisi economica e politica della Repubblica fa sentire il suo peso anche nelle quotidiane relazioni di "vicinato".

Il volume prosegue l'analisi delle molteplici identità ebraiche con l'interessante sezione dedicata alle Letterature. Attraverso saggi di analisi letteraria su scrittori e scrittrici ebrei del Novecento, quali Philip Roth, David Grossman e Zuzanna Ginczanka, gli autori descrivono tre ulteriori modalità dell'ebraicità moderna. Spostando il piano d'analisi sullo stato di Israele e su cosa vuol dire essere ebreo oggi, Massimiliano A. Boni nel suo "Una specie di distillato di umanità": Liidentità ebraica in Operazione Shylock di Philip Roth, descrive attraverso le pagine di questo romanzo un'identità plurima e le molte contraddizioni che gli ebrei incontrano nella definizione di loro stessi. Il romanzo di Roth, infatti, mette in evidenza diverse anime dell'essere ebreo e le molteplici questioni che solleva la loro definizione, a cominciare dal "problema" della diaspora, che è anche il filo rosso del romanzo. Tuttavia, al di là di ogni possibile fraintendimento, quello che Roth e Boni pongono in risalto è la "normalità" degli ebrei moderni, i quali appunto non vanno considerati dei “diversi”, degli altri da noi.

Il tema dell'identità e dei sentimenti degli ebrei viene ripreso in modo inedito anche nel saggio di Dario Miccoli, Le sue parole, le nostre voci: tradurre David Grossman tra Israele, Europa e Stati Uniti d'America. Qui il piano analitico si fa più letterario ma non meno pregnante e interessante. L'autore, riportando quanto emerso dalla tavola rotonda del convegno David Grossman and the Reception of Hebrew Literature in Europe and US - tenutosi a Venezia nel marzo 2011, organizzato da Emanuela Trevisan Semi e Vered Shemtov con la collaborazione delle Università Ca' Foscari e di Stanford ha descritto, da un lato, l'ampia ricezione dell'autore ebreo nel vecchio continente e negli USA e, dall'altro, ha espresso, attraverso le voci dei principali traduttori di Grossman, la difficoltà o la semplicità che essi hanno incontrato a trasmettere nelle loro lingue i sentimenti espressi nel romanzo, così come la scoperta di numerosi tratti lessicali comuni tra l'ebraico e le diverse lingue occidentali in cui il libro è stato tradotto. 
La terza parte si conclude con il saggio di Alessandro Amenta, Zuzanna Ginczanka: poetessa polacca e "bella ebrea", in cui si narrano le vicende della vita e della carriera poetica di una ragazza che sebbene sia nata da genitori ebrei si è sempre sentita polacca per cultura ed educazione. Il saggio, anche se può sembrare l'esempio più chiaro di un'assimilazione volontaria, si propone, al contrario, come la descrizione del lento cammino intrapreso da Zuzanna alla ricerca della sua identità.

Il volume si conclude con la parte dedicata all'Impero ottomano - Ebraismo nei territori ottomani - i cui saggi raccontano due aspetti della storia ebraica nel Vicino Oriente ottomano facendo luce sul ruolo che il millet ebraico ebbe all'interno del cosmopolita Impero d'Oriente sia a livello sociale che politico, ricostruendo anche alcuni insoliti legami con il nostro Paese.

Il primo saggio di Davide Saponaro, Sa'arot teman: tempeste messianiche nello Yemen ebraico, si occupa delle dinamiche sociali e locali dello sviluppo delle correnti messianiche yemenite tra il XVII e il XIX secolo, mettendo in evidenza come, nonostante una società fortemente stratificata e poco porosa nelle relazioni intercomunitarie, i movimenti messianici ebraici abbiano interessato e coinvolto anche le popolazioni musulmane locali, dimostrando come fossero dei movimenti trasversali e come entrambe le religioni fossero condizionate internamente da spinte di questo genere.

Il secondo saggio, Massoneria Italiana, ebraismo e movimento dei Giovani Turchi, sposta la sua attenzione dalla periferia al centro dell'Impero e in particolar modo alla regione macedone e stanbuliota. In una delle aree più "calde" dell'Impero alla fine del XIX secolo, Luca G. Manenti, analizza l'apporto dato dalle logge massoniche e dagli ebrei italiani allo sviluppo politico dei Giovani Turchi, movimento rivoluzionario sorto alla fine del XIX secolo e giunto al potere con la rivoluzione del 1908, e successivamente divenuto guida dell'Impero nel 1913 grazie al Comitato di Unione e Progresso (CUP). Il saggio di Manenti, evidenziando i legami strettissimi tra questo movimento e le logge massoniche della Penisola, riesce a far luce in modo chiaro su una pagina della nostra storia ancora poco nota al grande pubblico, mettendo in risalto il ruolo fondamentale giocato dagli ebrei in questa particolare relazione italo-turca. Un ruolo, come si vede dall'articolo, che non viene mai meno neanche in seguito all'occupazione italiana della Libia (1911), il punto più basso delle relazioni italo-turche. Di riflesso al ruolo svolto nei confronti dei Turchi, il saggio di Manenti sottolinea anche un altro fattore decisivo: il patriottismo ebraico verso il nostro Paese; un aspetto spesso dimenticato che, invece, l'autore fa risaltare chiaramente. 
Il volume qui presentato si avvale, accanto al dettagliato corpus di note dei singoli articoli, di un ottimo ed interessante apparato fotografico, il quale, sebbene le foto siano solo in bianco e nero, fornisce un importante contributo visivo alle "storie" narrate e dà un volto ai diversi personaggi descritti. Per queste ragioni, unitamente all'accuratezza dei saggi proposti, il volume LXXVIII della Rassegna mensile di Israel, si propone come un utile strumento per gli addetti ai lavori ed anche per il vasto pubblico interessato a scoprire e a capire in modo più chiaro le molteplici identità ebraiche e le diverse sfaccettature di una comunità molto spesso identificata come un monolite, semplificazione comoda per alcuni, ma non sufficiente ad indagare ed interagire con le differenti anime che la compongono. Il volume qui proposto, così come l'intera Rassegna mensile di Israel, infatti, cercano proprio di compiere un'opera di chiarificazione su queste realtà in modo da avvicinarci alla complessità e alla intrinseca bellezza della comunità ebraica, facendo anche emergere il ruolo decisivo che la comunità ebraica italiana ha avuto, in più occasioni, nel nostro Paese. 


\section{* L'autore}

Luca Zuccolo è dottore in Storia Contemporanea presso il SUM (Istituto Italiano di Scienze Umane) di Napoli. Già dottore magistrale in Storia d'Europa (Bologna, 2008), il suo campo di ricerca si rivolge allo sviluppo della modernità durante l'ultimo secolo dell'Impero Ottomano, al confronto/scontro tra modernità e tradizione in un contesto cosmopolita e allo sviluppo dei movimenti sociali che hanno preparato l'avvento della società turca contemporanea. È il referente di Diacronie per la storia turca e ottomana.

URL: < http://www.studistorici.com/2009/o2/24/jacopo_bassi/ >

\section{Per citare questo articolo:}

ZUCCOLO, Luca, «Recensione: UCEI (a cura di), Migrazioni, Memorie : La Rassegna Mensile di Israel, LXXVIII, 3/2012, Firenze, La Giuntina, 2013, 181 pp.», Diacronie. Studi di Storia Contemporanea : Le città di Babele, 29/3/2015,

URL:<http://www.studistorici.com/2015/03/29/zuccolo_numero_21/ >

\section{Diacronie Studi di Storia Contemporanea $\beta$ www.diacronie.it}

Risorsa digitale indipendente a carattere storiografico. Uscita trimestrale. redazione.diacronie@hotmail.it

Comitato di redazione: Jacopo Bassi - Luca Bufarale - Elisa Grandi - Deborah Paci - Fausto Pietrancosta - Matteo Tomasoni - Luca Zuccolo

Diritti: gli articoli di Diacronie. Studi di Storia Contemporanea sono pubblicati sotto licenza Creative Commons 2.5 . Possono essere riprodotti a patto di non modificarne i contenuti e di non usarli per fini commerciali. La citazione di estratti è comunque sempre autorizzata, nei limiti previsti dalla legge. 